\title{
ABOLIZIONE DELLE PROVINCE E RIALLOCAZIONE DELLE SPESE: IL CASO DELLA LOMBARDIA
}

Francesca Nordi, Università di Messina e Università di Ferrara

Leonzio Rizzo, Università di Ferrara e IEB Barcelona

JEL Classification: $\mathrm{H} 70, \mathrm{H} 72, \mathrm{H} 77$

Keywords: Legge 56/2014, risparmi di spesa, aree gestionali, province 


\title{
Abolizione delle province e riallocazione delle spese: il caso della Lombardia di Francesca Nordi *, Leonzio Rizzo
}

\begin{abstract}
La legge 56/2014 (cosiddetta legge Delrio), recepita dalla Regione Lombardia con legge regionale 19/2015, ha definito le funzioni di spesa fondamentali che devono rimanere in capo alle Province. Le restanti funzioni non fondamentali saranno, sempre secondo la legge, gestite dalle Regioni. In questo individuiamo le funzioni di spesa fondamentali confrontando quelle dei consuntivi provinciali con quelle definite dalla legge Delrio. Proponiamo quindi di quantificare il possibile risparmio confrontando le spese (rimaste in capo alle Province) pro-capite con quelle di Province simili dal punto di vista demografico. In particolare confrontiamo le spese pro-capite delle Province lombarde con le spese pro-capite medie nazionali delle rispettive classi di popolazione di appartenenza. Inoltre, il lavoro identifica aree dimensionali, alternative all'area circoscritta dall'intera Regione, di gestione efficiente delle funzioni non fondamentali che la legge 56/2014 delega alle Regioni.
\end{abstract}

JEL: H70, H72, H77

Parole chiave: Legge 56/2014, risparmi di spesa, aree gestionali, province

\footnotetext{
* Università di Messina \& Università di Ferrara, fnordi@ unime.it.

** Università di Ferrara \& IEB (Barcelona), rzzlzg@unife.it.
} 


\section{Introduzione}

La legge 56/2014 (d'ora in poi legge Delrio) introduce importanti modifiche nella gestione e allocazione delle funzioni di spesa delle Province italiane. Il primo effetto riguarda il nuovo assetto di governance istituzionale: gli organi politici non sono più eletti direttamente dalla cittadinanza, ma indirettamente dai Sindaci e dai Consiglieri dei comuni di appartenenza. Il secondo effetto riguarda la definizione delle funzioni fondamentali destinate a rimanere in capo alle Province e la definizione dei perimetri territoriali per le restanti funzioni.

La legge fissava al 31 dicembre 2014 il termine per l'approvazione delle leggi regionali di riordino delle funzioni, nel rispetto dei principi costituzionali di sussidiarietà, differenziazione e adeguatezza. A seguito del ritardo nell'esperimento di tali compiti, Governo, Regioni, Province e Comuni hanno siglato un Accordo in Conferenza Unificata (11 settembre 2014) modificando i tempi di approvazione delle leggi regionali. Nel corso del 2015 tutte le Regioni a statuto ordinario sono riuscite a normare la materia. Il Consiglio dei Ministri ha provveduto ad emanare il DPCM $^{1}$ del 26 settembre 2014 con i criteri generali di individuazione delle risorse da riallocare. All'interno del quadro di riforma delineato si inserisce la legge di stabilità per il $2015^{2}$ con le disposizioni di riduzione della spesa pubblica e la legge di stabilità $2016^{3}$ con quale sono concessi spazi di spesa alle province in ritardo nella riallocazione del personale eccedente le funzioni fondamentali.

Le funzioni fondamentali individuate dalla legge Delrio al comma 85 sono: a) pianificazione territoriale provinciale di coordinamento, nonché tutela e valorizzazione dell'ambiente, per gli aspetti di competenza; b) pianificazione dei servizi di trasporto in ambito provinciale, autorizzazione e controllo in materia di trasporto privato, in coerenza con la programmazione regionale, nonché costruzione e gestione delle strade provinciali e regolazione della circolazione stradale ad esse inerente; c) programmazione provinciale della rete scolastica, nel rispetto della programmazione regionale; d) raccolta ed elaborazione di dati, assistenza tecnico-amministrativa agli enti locali; e) gestione dell'edilizia scolastica; f) controllo dei fenomeni discriminatori in ambito occupazionale e promozione delle pari opportunità sul territorio provinciale. Queste funzioni difficilmente corrispondono a voci di bilancio specifiche.

Per le restanti funzioni, definite non fondamentali, l'Accordo in Conferenza Unificata attribuisce alla competenza dello Stato la materia di tutela delle minoranze ${ }^{4}$, e le restanti alla competenza delle Regioni, alle quali spetta per ogni singolo caso definirne l'elenco e operarne il riordino, ovvero stabilire quali funzioni demandare ad un livello di gestione inferiore (Comuni e loro forme associative).

La Regione Lombardia ha avviato il processo di riordino con legge regionale 8 luglio 2015, n. 19, trasferendo in capo alla Regione funzioni in passato conferite alle Province, nelle materie di agricoltura, foreste, caccia e pesca, ambiente ed energia. Esclusa dal riordino è la Provincia di Sondrio, individuata come titolare di specialità con riguardo alle caratteristiche geomorfologiche del territorio; la Città Metropolitana di Milano, invece, diviene titolare di ampie funzioni amministrative. Per le restanti funzioni in capo alle province la Regione tenta la sperimentazione di nuovi ambiti gestionali con l'istituzione di tavoli di confronto tra i diversi livelli di governo interessati; caso particolare spetta alla funzione nel campo dei trasporti.

Le Province lombarde presentano valori di spesa per le funzioni fondamentali in linea con i valori nazionali o sono possibili dei risparmi? In relazione alla riattribuzione delle funzioni non fondamentali qual è la dimensione ottimale per la gestione di quest'ultime?

\footnotetext{
${ }^{1}$ «Criteri per l'individuazione dei beni e delle risorse finanziarie, umane, strumentali e organizzative connesse con l'esercizio delle funzioni provinciali»

${ }^{2}$ Legge n.190/2014 comma 418 «Le province e le città metropolitane concorrono al contenimento della spesa pubblica attraverso una riduzione della spesa corrente di 1.000 milioni di euro per l'anno 2015, di 2.000 milioni di euro per l'anno 2016 e di 3.000 milioni di euro a decorrere dall'anno $2017 \ldots »$

${ }^{3}$ Legge n. 208/2015 comma 764

${ }^{4}$ Accordo tra Governo e Regioni in Conferenza Unificata 11 settembre 2014 p. 9. lettera b) lo Stato dichiara che rientra nelle proprie competenze il riordino delle seguenti funzioni amministrative, non riconducibili alle funzioni fondamentali di cui all'art. 1 , comma 85 della Legge, attualmente esercitate dalle Province.
} 
Per rispondere a queste domande utilizziamo un data-set comprensivo dei dati finanziari e demografici delle Province italiane, escluse le Province di Torino, Milano, Venezia, Genova, Bologna, Firenze, Bari, Napoli e Reggio Calabria, in quanto Città Metropolitane (così come stabilito dalla legge Delrio) e le Province appartenenti alle Regioni a statuto speciale (Sicilia, Sardegna, Friuli Venezia Giulia, Valle d'Aosta e Trentino Alto Adige).

\section{Quadro sintetico della spesa delle Province lombarde nel periodo 2000-2012}

Il peso delle spese provinciali lombarde è pari nel 2012 allo 0,49\% del Pil regionale: in termini assoluti si tratta di 1,64 miliardi di euro, di cui 1,16 miliardi per la spesa corrente e 476 milioni per la spesa in investimenti. La spesa delle Province presenta un trend decrescente a partire dall'anno 2003, guidato dall'andamento degli investimenti (fig. 1), che subiscono un drastico calo passando da 2,2 miliardi di euro nel 2003 al valore minimo di 397 milioni nel 2011.

La spesa corrente, al contrario, si mantiene sostanzialmente invariata fino al 2009, anno dopo il quale si registra una lieve diminuzione in valore assoluto pari a 220 milioni di euro (da 0,41\% nel 2009 a $0,35 \%$ sul PIL nel 2012), probabilmente oggetto di tagli a livello centrale dovuti alla crisi economica.

Fig. 1 - Andamento delle spese provinciali lombarde nel periodo 2000-2012.

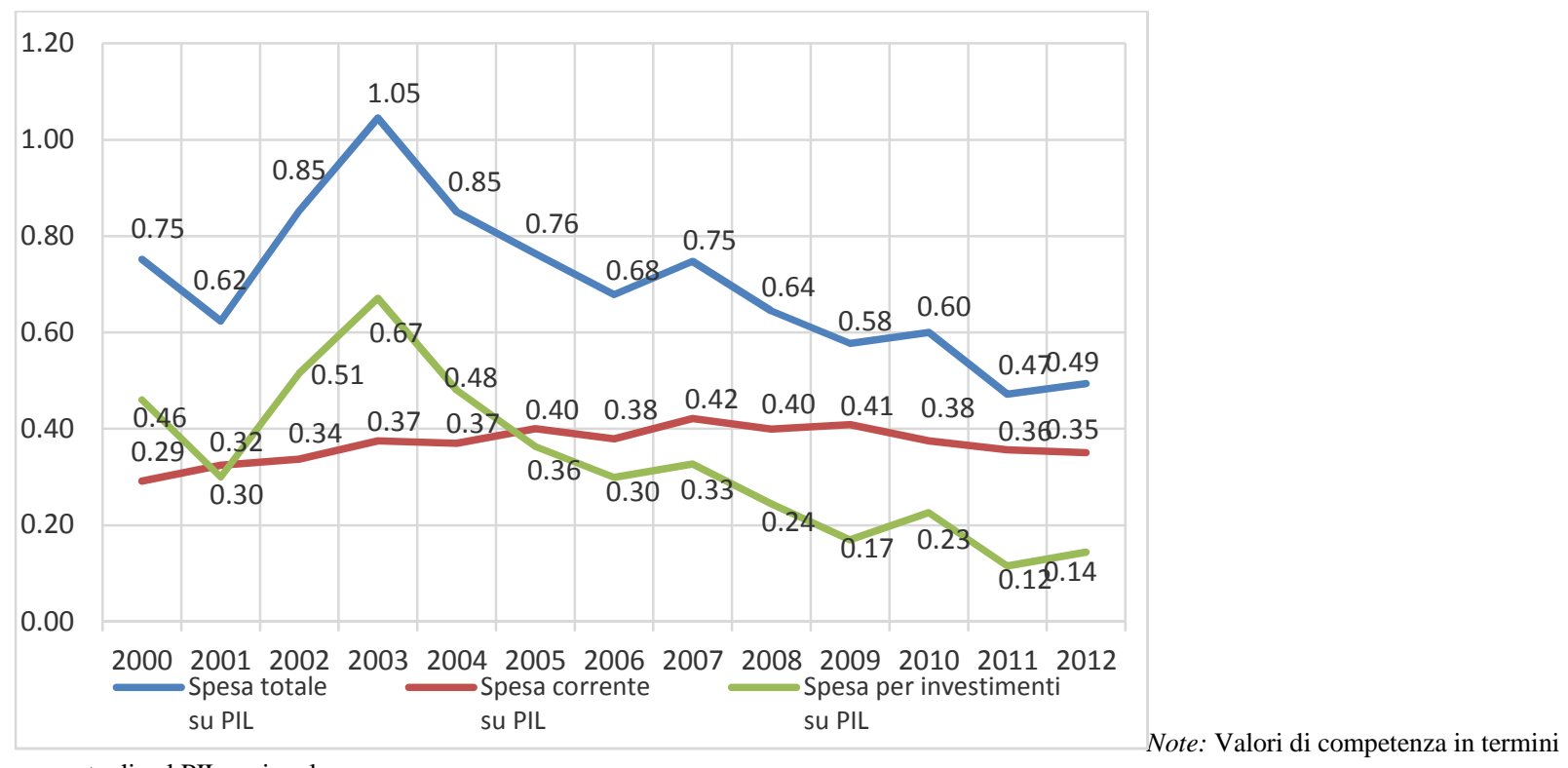

percentuali sul PIL regionale.

Le funzioni del bilancio che nel 2012 assorbono la maggior parte della spesa (tab.1) sono Amministrazione (26\%), Istruzione (14\%), Trasporti (16\%), Gestione del Territorio (22\%), Ambiente (9\%) e Sviluppo Economico $(7 \%)$. 
Tab. 1 - Funzioni provinciali.

\begin{tabular}{lllll}
\hline Funzione & Spesa corrente & Spesa c/capitale & Spesa totale & $\begin{array}{l}\text { Valore percentuale } \\
\text { spesa totale }\end{array}$ \\
\hline Amministrazione & 364 & 57 & 421 & 25.69 \\
Istruzione & 211 & 24 & 235 & 14.34 \\
Cultura & 23 & 10 & 33 & 2.00 \\
Turismo & 18 & 5 & 23 & 1.44 \\
Trasporti & 227 & 35 & 262 & 15.99 \\
Gestione territorio & 123 & 238 & 361 & 22.01 \\
Ambiente & 67 & 76 & 143 & 8.77 \\
Sociale & 37 & 2 & 39 & 2.39 \\
Sviluppo economico & 92 & 29 & 121 & 7.38 \\
\hline Totale & 1161 & 476 & 1637 & 100.00 \\
\hline
\end{tabular}

Note: Valori di competenza in milioni di euro e percentuale per l'anno 2012.

\section{La spesa di competenza delle Province secondo la nuova ripartizione della legge Delrio}

Non vi è una chiara corrispondenza tra le funzioni di spesa fondamentali individuate dalla legge Delrio e quelle che appaiono nei bilanci consuntivi delle Province. Dalla definizione delle voci di bilancio esistenti e da una ragionevole interpretazione delle funzioni definite dalla legge, abbiamo individuato una quota di spesa contenuta nei bilanci consuntivi, che può rientrare nel nuovo perimetro delle funzioni fondamentali delle province (tab. 2): a) la funzione Pianificazione territoriale provinciale di coordinamento, nonché tutela e valorizzazione dell'ambiente definita nella legge Delrio corrisponde alla somma di Servizio Urbanistica e programmazione territoriale e Servizio di tutela e valorizzazione ambientale del conto consuntivo della provincia; b) la funzione pianificazione dei servizi di trasporto in ambito provinciale, autorizzazione e controllo in materia di trasporto privato, in coerenza con la programmazione regionale, nonché costruzione e gestione delle strade provinciali e regolazione della circolazione stradale ad esse inerente definita nella legge Delrio, corrisponde alla Funzione nel campo dei trasporti sommata al Servizio Viabilità (che nell'attuale consuntivo si trova all'interno della funzione gestione del territorio), nella nostra analisi la Funzione trasporti sarà considerata separatamente, così come stabilito nel caso della regione Lombardia; c) programmazione provinciale della rete scolastica, nel rispetto della programmazione regionale definita nella legge Delrio, coincide con la spesa in conto corrente (del conto consuntivo della Provincia) di Servizio relativo agli Istituti di istruzione secondaria e Servizio relativo agli Istituti gestiti direttamente dalla Provincia; d) raccolta ed elaborazione di dati, assistenza tecnico-amministrativa agli enti locali così come definita nella legge Delrio è individuata da Servizio statistico e da Servizio di assistenza tecnico amministrativa agli Enti locali della Provincia, che nel consuntivo attuale si trovano entrambi nella funzione Amministrazione; e) la gestione dell'edilizia scolastica che appare nella legge Delrio coincide nel consuntivo della provincia con la spesa in conto capitale di Servizio relativo agli Istituti di istruzione secondaria e Servizio relativo agli Istituti gestiti direttamente dalla Provincia; f) infine abbiamo deciso di non considerare la funzione controllo dei fenomeni discriminatori in ambito occupazionale e promozione delle pari opportunità sul territorio provinciale, poiché non è possibile scorporarla dal Servizio mercato del lavoro, che si trova all'interno della Funzione sviluppo economico dei consuntivi provinciali.

La funzione amministrazione che rappresenta il $25 \%$ del totale (par. 5), è analizzata separatamente dalle altre funzioni. 
Tab. 2 - Tabella di raccordo delle funzioni fondamentali provinciali tra le funzioni elencate dalla legge Delrio e le voci di bilancio consuntivo provinciale.

\begin{tabular}{|c|c|c|}
\hline Funzione & Funzioni della legge Delio & $\begin{array}{l}\text { Funzioni elo Servizi dal bilancio consuntivo } \\
\text { provinciale }\end{array}$ \\
\hline 1 & $\begin{array}{l}\text { a) Pianificazione territoriale provinciale di } \\
\text { coordinamento, nonché tutela e valorizzazione } \\
\text { dell'ambiente }\end{array}$ & $\begin{array}{l}\text { Servizio Urbanistica e programmazione territoriale } \\
\text { Servizio di tutela e valorizzazione ambientale }\end{array}$ \\
\hline 2 & $\begin{array}{l}\text { b) Funzione pianificazione dei servizi di trasporto } \\
\text { in ambito provinciale, autorizzazione e controllo in } \\
\text { materia di trasporto privato, in coerenza con la } \\
\text { programmazione regionale, nonché costruzione e } \\
\text { gestione delle strade provinciali e regolazione della } \\
\text { circolazione stradale ad esse inerente }\end{array}$ & $\begin{array}{l}\text { La Funzione nel campo dei trasporti è oggetto di } \\
\text { analisi separata come disciplinato dalla l.r. 19/2015 } \\
\text { della Lombardia } \\
\text { Servizio Viabilità (all'interno della funzione } \\
\text { gestione del territorio) }\end{array}$ \\
\hline \multirow{2}{*}{3} & $\begin{array}{l}\text { c) Programmazione provinciale della rete } \\
\text { scolastica, nel rispetto della programmazione } \\
\text { regionale }\end{array}$ & $\begin{array}{l}\text { Spesa in conto corrente di Servizio relativo agli } \\
\text { Istituti di istruzione secondaria } \\
\text { Servizio relativo agli Istituti gestiti direttamente } \\
\text { dalla Provincia }\end{array}$ \\
\hline & e) Gestione dell'edilizia scolastica & $\begin{array}{l}\text { Spesa in conto capitale di Servizio relativo agli } \\
\text { Istituti di istruzione secondaria } \\
\text { Servizio relativo agli Istituti gestiti direttamente } \\
\text { dalla Provincia }\end{array}$ \\
\hline 4 & $\begin{array}{l}\text { d) Raccolta ed elaborazione di dati, assistenza } \\
\text { tecnico-amministrativa agli enti locali }\end{array}$ & $\begin{array}{l}\text { Servizio statistico } \\
\text { Servizio di assistenza tecnico amministrativa agli } \\
\text { Enti locali della Provincia (nella funzione } \\
\text { Amministrazione) }\end{array}$ \\
\hline Non considerata & $\begin{array}{l}\text { f) Funzione controllo dei fenomeni discriminatori in } \\
\text { ambito occupazionale e promozione delle pari } \\
\text { opportunità sul territorio provinciale }\end{array}$ & $\begin{array}{l}\text { Servizio mercato del lavoro (Funzione sviluppo } \\
\text { economico degli attuali consuntivi provinciali). }\end{array}$ \\
\hline
\end{tabular}

Secondo la classificazione precedentemente descritta (tab. 2) il valore della spesa per funzioni fondamentali (escludendo la funzione trasporto pubblico locale) è per la Lombardia 611 milioni di euro (tab.3), ovvero il $37,30 \%$ del totale. Le funzioni non fondamentali ammontano a 344 milioni di euro (21\%), e le spese generali di amministrazione sono 421 milioni di euro $(26 \%)$. La funzione trasporto pubblico locale è pari al $16 \%$ del totale delle spese per un valore di 262 milioni di euro. I valori percentuali rilevati in Lombardia sono simili a quelli riscontrati a livello nazionale, dove le funzioni fondamentali rappresentano il 37,7\% del totale, quelle non fondamentali il $23 \%$, le spese di amministrazione il $25 \%$ e le spese della funzione trasporti il $14,6 \%$.

Tab. 3 - Funzioni fondamentali secondo la classificazione di tabella 2.

\begin{tabular}{|c|c|c|c|c|}
\hline Funzioni & Spesa corrente & Spesa c/capitale & Spesa totale & $\begin{array}{l}\text { Valore percentuale } \\
\text { spesa totale }\end{array}$ \\
\hline 1 & 21 & 2 & 23 & 1.35 \\
\hline 2 & 116 & 238 & 354 & 21.60 \\
\hline 3 & 211 & 24 & 235 & 14.34 \\
\hline 4 & 0 & 0 & 0 & 0.01 \\
\hline Totale funzioni fondamentali & 347 & 263 & 611 & 37.30 \\
\hline Amministrazione & 364 & 57 & 421 & 25.68 \\
\hline Funzioni non fondamentali & 223 & 121 & 344 & 21.03 \\
\hline Funzione trasporto & 227 & 35 & 262 & 16 \\
\hline Totale & 1,161 & 476 & 1,637 & 100.00 \\
\hline
\end{tabular}




\section{Le funzioni di spesa fondamentali: un confronto tra Province lombarde e media delle Province italiane}

Al fine di comprendere i possibili risparmi per le funzioni fondamentali che rimangono in capo alle Province e quindi individuare la possibilità di ragionevoli tagli di risorse, confrontiamo la spesa pro-capite per funzioni fondamentali delle Province lombarde con la spesa pro-capite media delle Province italiane.

Le spese correnti di competenza ${ }^{5}$ (al netto della funzione trasporti) pro-capite delle funzioni fondamentali delle Province italiane presentano un andamento decrescente (cosiddetto ad L) rispetto alla popolazione (fig. 2).

Fig. 2 - Spesa corrente pro-capite fondamentale rapportata alla popolazione.

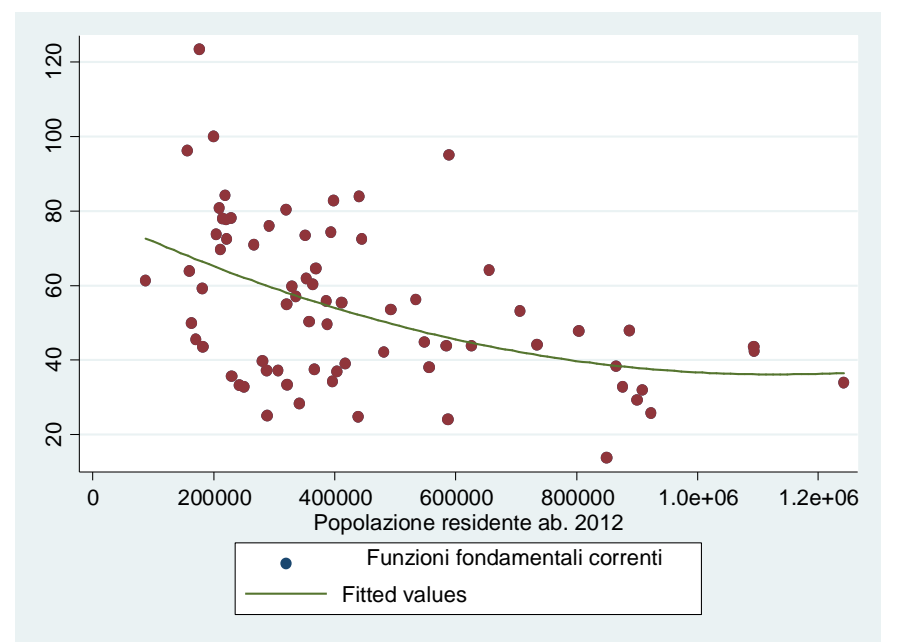

Note: Dati di competenza per le Province italiane anno 2012.

Individuiamo nel data-set sei classi (tab. 4) di popolazione all'interno delle quali analizzare le caratteristiche finanziarie delle Province.

Tab. 4 - Classi di popolazione e numero di Province per ciascuna classe demografica

\begin{tabular}{lll}
\hline Numero della classe & Classe di popolazione & Numero di Province per classe \\
\hline 1 & $0-210000$ & 12 \\
2 & $210000-310000$ & 15 \\
3 & $310000-420000$ & 20 \\
4 & $420000-805000$ & 16 \\
5 & $805000-1000000$ & 7 \\
6 & $1000000-1500000$ & 3 \\
\hline
\end{tabular}

\footnotetext{
${ }^{5} \mathrm{Si}$ è focalizzata l'analisi sulle spese correnti in quanto rappresentano il 70\% delle spese in termini di competenza delle amministrazioni provinciali e presentano un trend lineare nel periodo considerato.
} 
Utilizzando le classi di popolazione definite alla tab. 4, il valore minimo della spesa pro-capite corrente per funzioni fondamentali delle Province italiane si colloca nella classe di popolazione compresa tra 805.000 e 1.000.000 di abitanti. La media per la classe considerata è infatti di 31 euro pro-capite (tab. 5).

Tab - 5. Spesa media pro-capite corrente per funzioni fondamentali su classi di popolazione.

\begin{tabular}{lll}
\hline \multicolumn{2}{l}{ Classe di popolazione } & Spesa pro-capite fondamentale media \\
\hline 1 & $0-210000$ & 73 \\
2 & $210000-310000$ & 57 \\
3 & $310000-420000$ & 55 \\
4 & $420000-805000$ & 52 \\
5 & $805000-1000000$ & 31 \\
6 & $1000000-1500000$ & 40 \\
\hline \multicolumn{2}{l}{ Note: Dati di competenza per le Province italiane anno 2012. }
\end{tabular}

Le Province lombarde, escluso Bergamo e Varese, hanno spese pro-capite correnti minori rispetto alla media nazionale di classe. Si noti in particolare la Provincia di Lodi (appartenente alla classe di popolazione 2) con una spesa corrente pro-capite pari a 36 euro rispetto ad un valore nazionale di 57 ( -21 euro pro-capite) e quella di Lecco (classe di popolazione 3) con una spesa pro-capite inferiore di circa 27 euro rispetto al valore medio nazionale. Inoltre, la Provincia di Monza e della Brianza con 14 euro pro-capite, presenta il valore di spesa minimo della Regione.

Le funzioni fondamentali di spesa corrente associabili alle Province in seguito all'approvazione della legge Delrio, i cui valori sono riportati in tab. 6 (colonna 3 e/o 6), rappresentano l'ammontare di risorse storiche che le Province anche prima della riforma utilizzavano per adempiere alle funzioni di spesa identificate dalla stessa legge. 
Tab. 6 - Valori di spesa corrente fondamentale per le Province lombarde.

\begin{tabular}{|c|c|c|c|c|c|c|c|}
\hline Provincia & $\begin{array}{l}\text { Popolazione (in } \\
\text { migliaia) }\end{array}$ & $\begin{array}{l}\text { Classe di } \\
\text { Popolazione } \\
\text { (rif. Tab. 3) }\end{array}$ & $\begin{array}{l}\text { Spesa pro-capite } \\
\text { fondamentale }\end{array}$ & $\begin{array}{l}\text { Spesa media } \\
\text { nazionale }\end{array}$ & $\begin{array}{l}\text { Spesa standard } \\
\text { (milioni di euro) }\end{array}$ & $\begin{array}{l}\text { Spesa effettiva } \\
\text { (milioni di } \\
\text { euro) }\end{array}$ & $\begin{array}{l}\text { Risparmio di } \\
\text { spesa } \\
\text { (milioni di euro) }\end{array}$ \\
\hline & (1) & (2) & (3) & (4) & $(5)=\min \left[(1)^{*}(4),(1)^{*}(3)\right]$ & $(6)=(1) *(3)$ & $(7)=(6)-(5)$ \\
\hline SONDRIO & 181 & 1 & 59 & 73 & 11 & 11 & 0 \\
\hline LODI & 229 & 2 & 36 & 57 & 8 & 8 & 0 \\
\hline LECCO & 342 & 3 & 28 & 55 & 10 & 10 & 0 \\
\hline CREMONA & 358 & 3 & 50 & 55 & 18 & 18 & 0 \\
\hline MANTOVA & 417 & 3 & 39 & 55 & 16 & 16 & 0 \\
\hline PAVIA & 548 & 4 & 45 & 52 & 25 & 25 & 0 \\
\hline COMO & 587 & 4 & 24 & 52 & 14 & 14 & 0 \\
\hline MONZA.e BRIANZA & 850 & 5 & 14 & 31 & 12 & 12 & 0 \\
\hline VARESE & 888 & 5 & 48 & 31 & 28 & 43 & 15 \\
\hline BERGAMO & 1094 & 6 & 42 & 40 & 44 & 46 & 2 \\
\hline BRESCIA & 1242 & 6 & 34 & 40 & 42 & 42 & 0 \\
\hline Totale & 6736 & & & & 227 & 244 & 17 \\
\hline
\end{tabular}

Note: Dati di competenza anno 2012. 


\subsection{Una proposta di quantificazione dei risparmi sulle funzioni fondamentali}

In questo paragrafo proponiamo di quantificare il possibile risparmio confrontando le spese pro-capite di Province simili dal punto di vista demografico. Ovviamente le Province possono tra loro essere simili per altri fattori socio-economici, che determinano i fabbisogni di spesa, tuttavia l'elemento demografico è sicuramente di rilievo nello spiegare la variabilità della spesa (fig. 2). In particolare confrontiamo le spese pro-capite delle Province lombarde con le spese pro-capite medie nazionali delle rispettive classi di popolazione di appartenenza. Se la spesa pro-capite di una Provincia è inferiore al valore medio nazionale associato alla classe demografica di appartenenza si riconosce a tale Provincia la sua spesa storica, se invece la spesa pro-capite di una Provincia è superiore al valore medio nazionale associato alla classe demografica di appartenenza, si riconosce alla Provincia il valore medio della propria classe demografica e per determinare l'effettiva spesa complessiva ammissibile, si moltiplica tale valore per la popolazione della Provincia considerata.

Con questa procedura le Province di Lodi, Lecco, Cremona, Mantova, Pavia, Como, Monza e Brianza, e Brescia, continuano a spendere quanto spendevano in funzioni fondamentali prima della riforma, mentre le Province di Bergamo e Varese spenderebbero meno di quanto facevano prima della riforma ed in particolare Bergamo che prima aveva una spesa per funzioni fondamentali di 46 milioni spenderebbe dopo la riforma 44 milioni e Varese che prima aveva una spesa per funzioni fondamentali di 43 milioni spenderebbe dopo la riforma 28 milioni.

In aggregato il risparmio (tab. 4, colonna 7) sarebbe pari a circa 17 milioni di euro, ovvero ogni residente delle Province interessate otterrebbe in media circa 9 euro in meno di spesa per funzioni fondamentali ${ }^{6}$

È importante inoltre sottolineare che la spesa provinciale è spesso frutto di delega regionale e quindi nel confronto tra le performance delle varie Province, che appartengono a differenti Regioni è opportuno valutare se l'entità delle deleghe che le Regioni fanno alle proprie Province è molto differente.

\footnotetext{
${ }^{6}$ Ovviamente riforme del genere vanno implementate con estrema gradualità, ipotizzando dei riparti di risorse intermedi, che riconoscano in parte la spesa storica e che quest'ultimo fattore sia sempre meno incisivo durante un definito periodo di transizione.
} 
Tab. 7 - Valori di entrata pro-capite.

\begin{tabular}{ll}
\hline Provincia & $\begin{array}{l}\text { Contributi e trasferimenti } \\
\text { correnti dalla Regione } \\
\text { per funzioni delegate }\end{array}$ \\
\hline SONDRIO & 0 \\
LODI & 4 \\
LECCO & 6 \\
CREMONA & 4 \\
MANTOVA & 0 \\
PAVIA & 10 \\
COMO & 2 \\
MONZA E BRIANZA & 0 \\
VARESE & 0 \\
BERGAMO & 1 \\
BRESCIA & 3 \\
\hline Media delle Province lombarde & 3 \\
\hline Media nazionale & 31 \\
\hline Note: Dati di competenza anno 2012. &
\end{tabular}

Nel nostro caso partendo dai dati finanziari di bilancio è possibile rilevare le risorse che le Province ricevono dalla Regione per il finanziamento delle funzioni di spesa delegate (tab.7). Nel 2012 le Province di Sondrio, Mantova, Monza e Brianza e Varese non ricevono di fatto risorse per funzioni delegate. Il valore più elevato di trasferimenti per funzioni delegate è registrato da Pavia che riceve dalla Regione 10 euro pro-capite. In media la Regione Lombardia trasferisce alle Province per la gestione delle funzioni delegate 3 euro pro-capite, mentre a livello nazionale le Regioni trasferiscono in media 31 euro pro-capite.

Questo dato ci induce ad interpretare con una certa cautela le conclusioni a cui siamo giunti in questa sezione, dato che le Province italiane sono in media oberate da una spesa per funzioni delegate molto più elevata che le Province lombarde.

Questa preliminare proposta di quantificazione dei risparmi può essere sicuramente migliorata tenendo conto in una successiva analisi di alcuni importanti fattori. Dalla semplice media per classi demografiche si potrebbe passare ad utilizzare il valore interpolato di una regressione che tenga conto oltre che del fattore demografico anche di altri indicatori di tipo socio-economico. Un aspetto importante è anche quello dell'effettiva erogazione di servizi e relativa qualità, corrispondenti alla spesa per funzioni fondamentali, ottenibile tramite opportune e puntuali stime (Brunello, Porcelli e Stradiotto, 2015): il dato finanziario sarebbe in questo caso integrato con indicatori di qualità e quantità del servizio offerto quando disponibili.

\section{La dimensione ottimale per le funzioni non fondamentali}

Le funzioni non fondamentali sono oggetto di riattribuzione a Regioni, Comuni e/o Associazioni di Comuni.

Qual è l'area ottimale entro cui le funzioni non fondamentali devono essere gestite? Tale questione assume rilevanza per le Regioni, il cui ruolo consiste nell'identificare normativamente, le forme ottimali di gestione di questo tipo di funzioni.

Se dalle spese correnti totali delle Province sottraiamo le spese correnti che finanziano le funzioni fondamentali (individuate alla tabella 2 del paragrafo 3) e la funzione Amministrazione, otteniamo l'ammontare di 
spese correnti che finanziano le funzioni non fondamentali. Queste sono Cultura e beni culturali, Settore turistico, sportivo e ricreativo, Settore del sociale e parte delle funzioni Sviluppo economico e Tutela ambientale.

Le spese pro-capite correnti non fondamentali delle Province italiane presentano un andamento decrescente rispetto alla popolazione. (fig. 3).

Fig. 3 - Spesa corrente pro-capite non fondamentale rapportata alla popolazione. Dati di competenza per le Province italiane anno 2012.

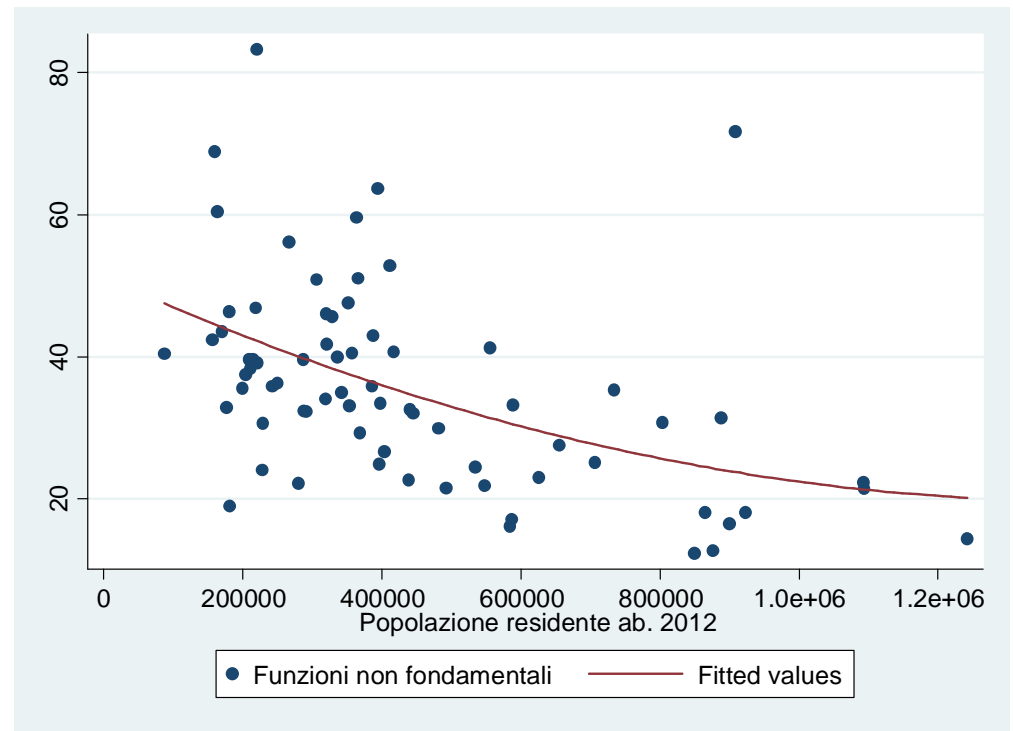

Note: Dati di competenza anno 2012.

In particolare il minimo della spesa pro-capite per funzioni non fondamentali, si trova nella classe di popolazione 6 (tab. 8), ovvero per gli enti con popolazione compresa tra 1 e 1,5 milioni di abitanti, che nel nostro caso sono Bergamo, Brescia e Salerno su un totale di 76 Province. Il valore della spesa per tale classe è pari a 19 euro pro-capite. Le classi delle Province con popolazione compresa tra 420 mila e 1 milione di abitanti (21 Province su un totale 76 ) hanno un valore medio che oscilla tra 27 e 26 euro pro-capite.

Tab. 8 - Spesa media pro-capite corrente per funzioni non fondamentali sulle classi di popolazione.

\begin{tabular}{lll}
\hline \multicolumn{2}{c}{ Classe di popolazione } & $\begin{array}{l}\text { Spesa pro-capite } \\
\text { non fondamentale media }\end{array}$ \\
\hline 1 & $0-210000$ & 42 \\
2 & $210000-310000$ & 40 \\
3 & $310000-420000$ & 41 \\
4 & $420000-805000$ & 27 \\
5 & $805000-1000000$ & 26 \\
6 & $1000000-1500000$ & 19 \\
\hline \multicolumn{2}{l}{ Note: Dati di competenza per le Province italiane anno 2012 }
\end{tabular}

Da questa analisi sembrerebbe opportuno che le funzioni non fondamentali siano svolte da enti di grandi dimensioni che ricomprendano popolazioni superiori a $1.000 .000 \mathrm{di}$ abitanti. Per tali funzioni bisognerebbe ricostruire un perimetro simile a quello delle Città Metropolitane. La gestione delle funzioni non fondamentali 
potrebbe quindi essere affidata alle Regioni, piuttosto che a forme associative di Comuni che difficilmente coprirebbero le dimensioni demografiche che superano il milione di abitanti.

In una Regione come la Lombardia ad esempio la gestione della funzione turistica potrebbe all'interno della Regione essere gestita con presidi regionali sul territorio per aree demografiche di 1.500 .000 abitanti: ciò significherebbe suddividere la Lombardia in 6/7 aree, ovvero accorpare le Province esistenti su perimetri che permettono di gestire le funzioni considerate in modo più efficiente. Un'alternativa di second best potrebbe essere quella di continuare a lasciar gestire le spese non fondamentali alle Province di Bergamo e Brescia e destinare alla Regione la gestione delle spese non fondamentali per le Province con popolazione inferiore ad $1.000 .000 \mathrm{di}$ abitanti.

La legge regionale della Lombardia (1. r. n. 19/2015), in merito, ha previsto la creazione di tavoli istituzionali di confronto, «al fine di indicare, in via sperimentale e in accordo con la Regione, le zone omogenee eventualmente individuate dagli statuti provinciali quali ambiti territoriali ottimali per lo svolgimento in forma associata (...) di specifiche funzioni e servizi». Tale disposizione è il primo passo verso la configurazione degli ambiti gestionali ottimali, così come discusso in questo paragrafo.

\section{La funzione generale di amministrazione, gestione e controllo}

All'interno della funzione generale di amministrazione, gestione e controllo confluiscono le spese che l'ente effettua per garantire il funzionamento della propria macchina amministrativa. Nelle Province lombarde la spesa di amministrazione nel 2012 è pari al 26\% del totale, in linea con il livello medio nazionale (25\%). La spesa procapite delle Province italiane presenta un andamento decrescente rispetto alla popolazione, che si appiattisce dopo 800.000 abitanti (fig. 4).

Fig. 4 - Spesa corrente pro-capite di amministrazione rapportata alla popolazione. Dati di competenza per le Province italiane anno 2012.

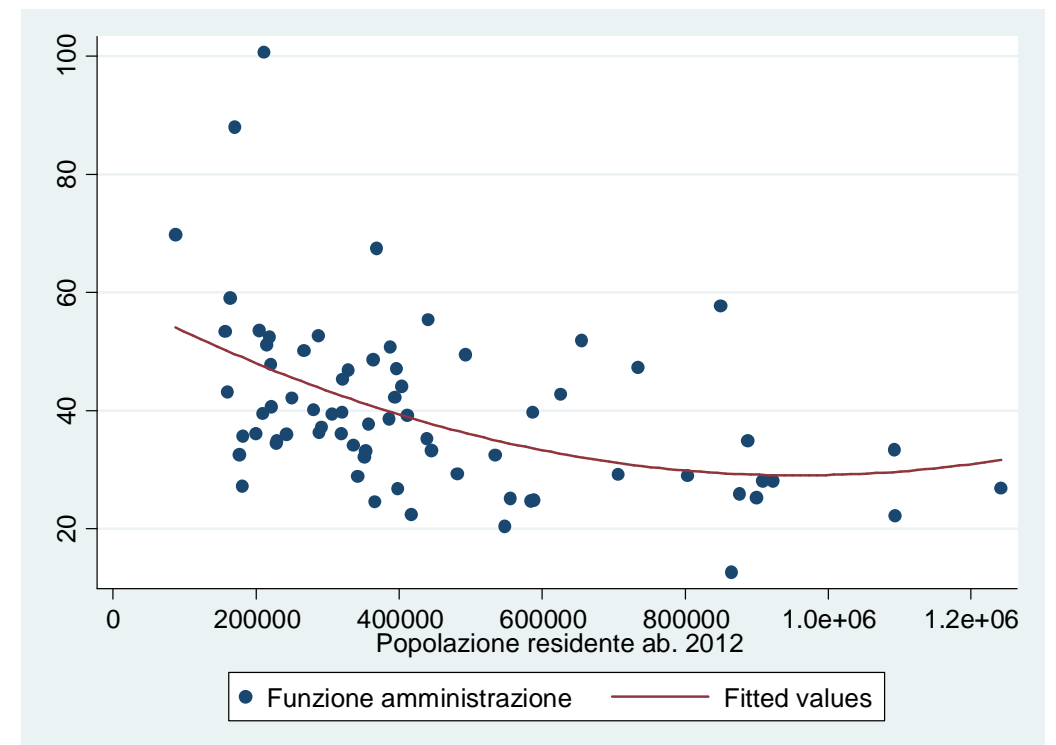

Note: Dati di competenza per le Province italiane anno 2012

A livello nazionale la classe di popolazione che presenta il valore minimo è quella compresa tra $1 \mathrm{e} 1,5$ milioni di abitanti con una spesa pro-capite di 27 euro. 
Tab. 9 - Spesa media pro-capite corrente per funzioni di amministrazione sulle classi di popolazione.

\begin{tabular}{lll}
\hline \multicolumn{2}{c}{ Classe di popolazione } & $\begin{array}{l}\text { Spesa pro-capite } \\
\text { amministrazione media }\end{array}$ \\
\hline 1 & $0-210000$ & 49 \\
2 & $210000-310000$ & 46 \\
3 & $310000-420000$ & 39 \\
4 & $420000-805000$ & 36 \\
5 & $805000-1000000$ & 30 \\
6 & $1000000-1500000$ & 27 \\
\hline
\end{tabular}

Note: Dati di competenza per le Province italiane anno 2012

Le Province lombarde presentano valori pro-capite di spesa corrente per amministrazione inferiori ai valori nazionali calcolati per la medesima classe di popolazione, se si eccettuano i casi di Como, Monza-Brianza e Varese (tab.10).

Tab. 10 - Valori di spesa corrente amministrazione per le Province lombarde.

\begin{tabular}{lllll}
\hline Provincia & Popolazione (in migliaia) $)$ & $\begin{array}{l}\text { Classe di } \\
\text { Popolazione } \\
\text { (rif. Tab. } 7)\end{array}$ & $\begin{array}{l}\text { Spesa pro-capite } \\
\text { amministrazione }\end{array}$ & Spesa media nazionale \\
\hline SONDRIO & 181 & 1 & 27 & 49 \\
LODI & 229 & 2 & 35 & 46 \\
LECCO & 342 & 3 & 29 & 39 \\
CREMONA & 358 & 3 & 38 & 39 \\
MANTOVA & 417 & 3 & 22 & 39 \\
PAVIA & 548 & 4 & 20 & 36 \\
COMO & 587 & 4 & 40 & 36 \\
MONZA E BRIANZA & 850 & 5 & 58 & 30 \\
VARESE & 888 & 5 & 35 & 30 \\
BERGAMO & 1094 & 6 & 22 & 27 \\
BRESCIA & 1242 & 6 & 27 & 27 \\
\hline
\end{tabular}

Note: Dati di competenza anno 2012

Si noti che nel caso, come quello finora discusso, di spostamento di funzioni ad altri enti si realizza una riduzione della complessità di gestione per l'ente dal quale le funzioni non amministrative migrano e sarebbe naturale una relativa riduzione anche delle risorse dedicate alla funzione dell' amministrazione. Al contrario per gli enti a cui vengono attribuite nuove funzioni la complessità aumenta e di conseguenza il fabbisogno di risorse correlate all'amministrazione. È necessario, magari in un approfondimento successivo a questo lavoro, costruire dei ragionevoli criteri di riallocazione della spesa per amministrazione che tengano conto della riallocazione delle funzioni non fondamentali. Su questo tema, infatti, la legge regionale della Lombardia (l. r. n. 19/2015), che recepisce il DPCM del 26 settembre 2014 sui criteri di allocazione delle risorse, prevede che la Giunta adotti "le disposizioni necessarie all'effettivo trasferimento delle funzioni (...) e l'individuazione e il trasferimento delle risorse umane, strumentali e finanziarie connesse alle funzioni oggetto di trasferimento". 


\section{Il caso lombardo della funzione di trasporto pubblico locale}

Un caso particolare è quello previsto per la funzione di trasporto pubblico locale. ${ }^{7}$ La Regione Lombardia, sfruttando il ruolo di programmatore regionale per la gestione del Servizio di trasporto ha previsto la suddivisione della Regione in sei bacini territoriali ottimali e omogenei, che sono: 1. Provincia di Bergamo, 2. Provincia di Brescia, 3. Province di Como, Lecco e Varese, 4. Province di Cremona e Mantova, 5. Città metropolitana di Milano, Province di Monza e Brianza, Lodi e Pavia, 6. Provincia di Sondrio.

Per calcolare i potenziali risparmi successivi alla definizione delle aree territoriali che in Lombardia dovranno gestire la funzione trasporti utilizziamo il data-set delle Province includendo anche le Città Metropolitane e quindi aggiungendo un'ulteriore fascia demografica: quella che comprende le aree con più di 1.500 .000 abitanti.

A livello nazionale, il valore minimo di spesa corrente per la funzione dei trasporti ricade nella classe di popolazione 4 (420.000-805.000 abitanti) con un valore medio pari a 22 euro pro-capite (tab. 12) se si escludono le Città Metropolitane. Se si includono anche le Città Metropolitane il valore minimo ricade nell'ultima classe demografica (quella delle città con più di 1.500 .000 abitanti), pari a 17 euro pro-capite (tab. 12).

\footnotetext{
${ }^{7}$ La funzione di trasporto pubblico locale nella legge Delrio è così definita: "pianificazione dei servizi di trasporto in ambito provinciale, autorizzazione e controllo in materia di trasporto privato, in coerenza con la programmazione regionale, nonché costruzione e gestione delle strade provinciali e regolazione della circolazione stradale ad esse inerente, rientra nelle funzioni fondamentali delle province."
} 
Tab. 12 - Spesa media pro-capite corrente per funzione trasporto su classi di popolazione.

\begin{tabular}{llll}
\hline \multicolumn{2}{c}{ Classe di popolazione } & Numero di Province & $\begin{array}{l}\text { Spesa pro-capite trasporti media } \\
\text { Tutte le Province }\end{array}$ \\
\hline 1 & $0-210000$ & 12 & 31 \\
2 & $210000-310000$ & 15 & 45 \\
3 & $310000-420000$ & 20 & 24 \\
4 & $420000-805000$ & 17 & 22 \\
5 & $805000-1000000$ & 10 & 29 \\
6 & $1000000-1500000$ & 5 & 24 \\
7 & Oltre 1500000 & 5 & 17 \\
\hline
\end{tabular}

Note: Dati di competenza per le Province italiane anno 2012. Sono incluse le Città Metropolitane ed escluse le Regioni a statuto speciale.

Utilizzando i valori medi di spesa per le Province italiane abbiamo calcolato i risparmi ottenibili dalla riallocazione della funzione trasporti effettuata sui bacini territoriali ottimali e omogenei definiti dalla Regione Lombardia. Abbiamo calcolato la spesa pro-capite delle nuove aree (colonna 4 tab. 13) dividendo la spesa effettiva - ottenuta sommando la spesa delle Province appartenenti ai differenti bacini - per il totale della popolazione residente nel nuovo aggregato. Successivamente confrontiamo le spese pro-capite delle aree lombarde con le spese pro-capite medie nazionali delle rispettive classi di popolazione di appartenenza. Se la spesa pro-capite di area è inferiore al valore medio nazionale associato alla classe demografica di appartenenza si riconosce a tale area la sua spesa storica, se invece la spesa pro-capite dell'area è superiore al valore medio nazionale associato alla classe demografica di appartenenza, allora gli si riconosce il valore medio della propria classe demografica e per determinare l'effettiva spesa complessiva ammissibile, si moltiplica tale valore per la popolazione del bacino territoriale provincia considerato.

Tutte le aree presentano una spesa pro-capite per trasporti superiore a quella nazionale (colonne $4-5$ ). Infatti la spesa standard riconosciuta all'area sarebbe sempre inferiore a quella effettiva. In termini aggregati il risparmio di spesa possibile, calcolato come differenza tra la spesa standard e la spesa effettiva sarebbe pari a 48 milioni di euro, ovvero in termini pro-capite a 7 euro per ogni cittadino lombardo (escludendo la Città Metropolitana di Milano).

Quindi la riorganizzazione della gestione della funzione trasporti per la Regione Lombardia potrebbe portare risparmi rilevanti (dall'analisi è esclusa la Città Metropolitana di Milano); ovviamente il risultato di questo esercizio necessita di ulteriori verifiche che permettano di confrontare non solo il dato finanziario, ma l'effettiva erogazione di servizi e relativa qualità, a parità di spesa. 
Tab. 12 - Valori di spesa corrente per la funzione di trasporto per le aree ottimali identificatedalla riorganizzazione prevista dalla l. r. 19/2015.

\begin{tabular}{|c|c|c|c|c|c|c|c|}
\hline Nuove aree & Popolazione (in migliaia) & $\begin{array}{l}\text { Classe di } \\
\text { popolazione } \\
\text { (Rif. Tab. 11) }\end{array}$ & $\begin{array}{l}\text { Spesa effettiva } \\
\text { (milioni di euro) }\end{array}$ & $\begin{array}{l}\text { Spesa pro-capite } \\
\text { trasporti }\end{array}$ & $\begin{array}{l}\text { Spesa media } \\
\text { nazionale }\end{array}$ & $\begin{array}{l}\text { Spesa standard } \\
\text { (milioni di euro) }\end{array}$ & $\begin{array}{l}\text { Risparmio di spesa } \\
\text { (milioni di euro) }\end{array}$ \\
\hline & (1) & (2) & (3) & $(4)=(3) /(1)$ & (5) & $(6)=(1) *(4)$ & $(7)=(6)-(5)$ \\
\hline SONDRIO & 181 & 1 & 7 & 40 & 31 & 6 & 2 \\
\hline CREMONA - MANTOVA & 775 & 4 & 26 & 34 & 22 & 17 & 9 \\
\hline BERGAMO & 1094 & 6 & 35 & 32 & 24 & 26 & 9 \\
\hline BRESCIA & 1242 & 6 & 34 & 28 & 24 & 30 & 5 \\
\hline $\begin{array}{l}\text { MONZA E BRIANZA - LODI - } \\
\text { PAVIA }\end{array}$ & 1627 & 7 & 45 & 27 & 17 & 28 & 17 \\
\hline COMO - LECCO - VARESE & 1817 & 7 & 38 & 21 & 17 & 31 & 7 \\
\hline Totale & 6735 & & 185 & & & 137 & 48 \\
\hline
\end{tabular}

Note: Dati di competenza anno 2012. 


\section{Conclusioni}

L'analisi condotta in questo articolo fornisce alcune indicazioni rilevanti per la riorganizzazione delle funzioni provinciali, secondo i principi stabiliti dalla legge Delrio. Nel lavoro sono stati utilizzati i dati finanziari delle Province italiane.

Per le funzioni fondamentali di spesa (come definite dalla legge Delrio) la classe di popolazione con il minimo di spesa è quella corrispondente all'intervallo che va da 805.000 a 1.000 .000 di abitanti; per le funzioni di spesa non fondamentali, la classe con il valore minimo di spesa è quella identificata dall'intervallo che va da 1 a 1,5 milioni di abitanti.

Abbiamo suddiviso l'analisi in due ambiti principali: risparmi ottenibili sulle funzioni fondamentali che rimangono in capo alle Province e ambiti ottimali di gestione delle funzioni non fondamentali che devono essere riallocate ad aree territoriali da definire.

Per le funzioni di spesa fondamentali nella Regione Lombardia sono possibili risparmi pari 17 milioni di euro. Tale valore è calcolato imponendo alle Province lombarde con valori di spesa pro-capite superiori a quelli medi nazionali della classe di popolazione di appartenenza, il valore della spesa pro-capite di quest'ultima.

Abbiamo discusso come le funzioni non fondamentali, in Lombardia, dovrebbero essere gestite su territori di dimensione almeno pari 1.500.000 abitanti: è quindi necessario un accorpamento delle Province esistenti su perimetri che permettano di gestire le funzioni non fondamentali in modo più efficiente.

Infine la gestione del servizio di trasporto pubblico allocata sul territorio lombardo attraverso gestioni associate, secondo la nostra simulazione, potrebbe portare a risparmi importanti pari a 48 milioni di euro, corrispondenti al 25\% della spesa attuale, escludendo la Città Metropolitana di Milano. 


\section{Bibliografia}

Brunello G., Porcelli F. e Stradiotto M. (2015). I fabbisogni standard dei comuni e delle province: un nuovo patrimonio informativo che potrebbe stimolare efficienza e responsabilità nei governi locali. Siep Working paper N. 685

Ferraresi M., Rizzo L. (2012). Fabbisogni e costi nella stima della spesa standard: una simulazione per i comuni pugliesi. Politica Economica, 3: 343-369. DOI: 10.1429/74178

Giarda, P. (1968). Spese ed imposte degli enti locali: verifica empirica di un modello di comportamento. Rivista di Diritto e Scienza delle Finanze, Anno XXVII, n. 4.

Porcelli F., Settimi C. e Stradiotto M. (2015). Cosa cambia con il debutto dei tagli non lineari. Lavoce.info. Testo disponibile al sito: http://www.lavoce.info/archives/34606/cosa-cambia-con-il-debutto-dei-tagli-non-lineari/ 